\title{
Efficiency Estimation of Energy Introduction of a Ultrasound Field Into the Zone of Drilling of Complex Packages From Composite Materials and Titanium
}

\author{
Evgeniy Kiselev ${ }^{1, *}$, Kirill Savelev ${ }^{1}$ and Oleg Krupennikov ${ }^{1}$ \\ ${ }^{1}$ Ulyanovsk State Technical University, 432027, 32 Severny Venets Street, Ulyanovsk, Ulyanovsk \\ region, the Russian Federation
}

\begin{abstract}
The possibility of improving the quality of holes in complex packages made of composite materials and titanium alloys in the drilling operation by using the energy of ultrasonic vibrations introduced into the treatment zone is considered. The construction of complex packages and the problems arising from their processing with an axial tool are described. The rational design of the experimental setup for testing the technology of ultrasonic drilling of small-diameter holes was chosen. The unit is based on the KUKA KR 16 R2100 robotic complex. In addition to the industrial robot, it contains a spindle attached to the robot; Ultrasonic head, dynamometer and table, on which the package made of composite materials and titanium alloys is fixed. Ultrasonic oscillations with a frequency of $22 \mathrm{kHz}$ can be transmitted, depending on the execution of the installation, either to the spindle or to the table with a complex package. The device allows carrying out experiments with the imposition of unmodulated, amplitude-modulated or frequency-modulated ultrasonic vibrations on the drill or on the object table with a package of composite materials.
\end{abstract}

\section{Introduction}

Currently, in the aviation industry both in Russia and abroad, there is a tendency to increase the share of use of composite materials (CM) in various structural elements of aircraft [1]. The use of $\mathrm{CM}$ is the only possible way to reduce the mass of the structure and improve flight performance, which contributes to reducing fuel consumption and increasing the payload of aircraft. At the same time, parts from CM of various grades are mated with structural elements of aluminum and (or) titanium alloys, forming complex packages. As a rule, parts of the power skeleton of the airframe and bypassing elements are made of aluminum and titanium alloys. The number of dissimilar materials in packages usually does not exceed three (titanium alloy, aluminum alloy, KM), and the total number of layers can reach five or more.

\footnotetext{
*Corresponding author: kec.ulstu@,mail.ru
} 
The composition of a complex package and the sequence of arrangement of layers depends on the design features and the purpose of the parts to be joined. Overwhelmingly, the joints are made by installing the connecting elements in the pre-machined holes. Since the constituent elements of such multilayer packages have different physico-mechanical properties (for example, low thermal conductivity and tendency to adhesion of titanium alloys, pronounced anisotropy of CM properties), therefore, in the process of their drilling, the following defects appear: delamination, destruction, uncut fibers, tears, melting of the polymer matrix, changing the shape of the holes, their breakdown, the formation of burns on the treated surfaces, etc. In turn, a decrease in the quality of the holes can lead to high contact loads, a decrease in the resource and the destruction of the joints in complex packages.

\section{Description of the experimental setup}

The Regional Technological Center for Industrial Internet in Mechanical Engineering and the Department of Mechanical Engineering Technology UISTU has accumulated considerable experience in intensifying mechanical processing processes using the energy of ultrasonic vibrations [2], which the authors proposed to use in drilling holes in complex packages.

For these purposes, in the regional technological center of the industrial Internet in mechanical engineering at UISTU, the authors created an experimental installation, the general view of which is presented in the figure 1 .

The unit was developed on the basis of the industrial robot KUKA KR 16 R2100.

As it is known, the processing of holes in complex packages can be performed on robotic complexes using the following schemes [3-6]:

1. Processing using a spindle assembly, providing only the rotational movement of the drill. Here the feed is carried out by the axes of the robot. With this method, the spindle assembly has minimal dimensions and weight, which contributes to work in hard-to-reach places and allows the use of a robot with a low carrying capacity. Consequently, this scheme can be used in practice for drilling holes of small diameter with the help of precision robots with a low carrying capacity.

2. The use of a special spindle assembly, providing both the rotation of the tool and its supply. In this case, the axes of the robot perform only the positioning of the working tool relative to the workpiece, the machining accuracy increases, however, the loads associated with the cutting process are also transmitted to the robot nodes. At the same time for the implementation of this scheme a special spindle is required, which has a more complex design and more weight, and hence a higher cost. In addition, to raise the heavy spindle, you need to use a high-capacity robot, which also leads to an increase in the cost of implementing this processing circuit.

3. Processing holes by orbital drilling, in which a special milling cutter is used as a cutting tool, the diameter of which is $60-80 \%$ of the diameter of the resulting hole.

The use of this processing scheme can significantly reduce cutting forces in the tool feed direction. At the same time, the possibility of practical application of this scheme is limited to the processing of large-diameter holes, since with small hole diameters (small cutter diameter), the tool's resistance to spinning decreases, which leads to a deterioration in the quality of the holes produced and may contribute to rapid tool breakage.

In view of the above, the authors developed the first layout of the robotic complex in the developed experimental setup for testing the technology of ultrasonic drilling of smalldiameter holes. 


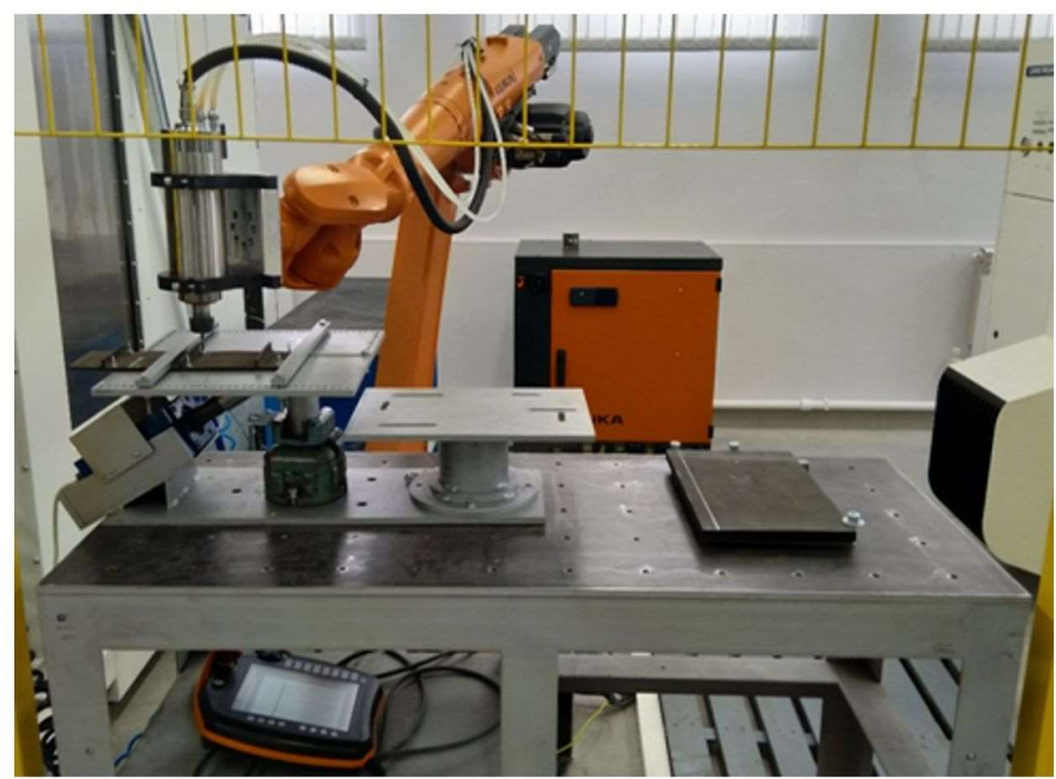

a)

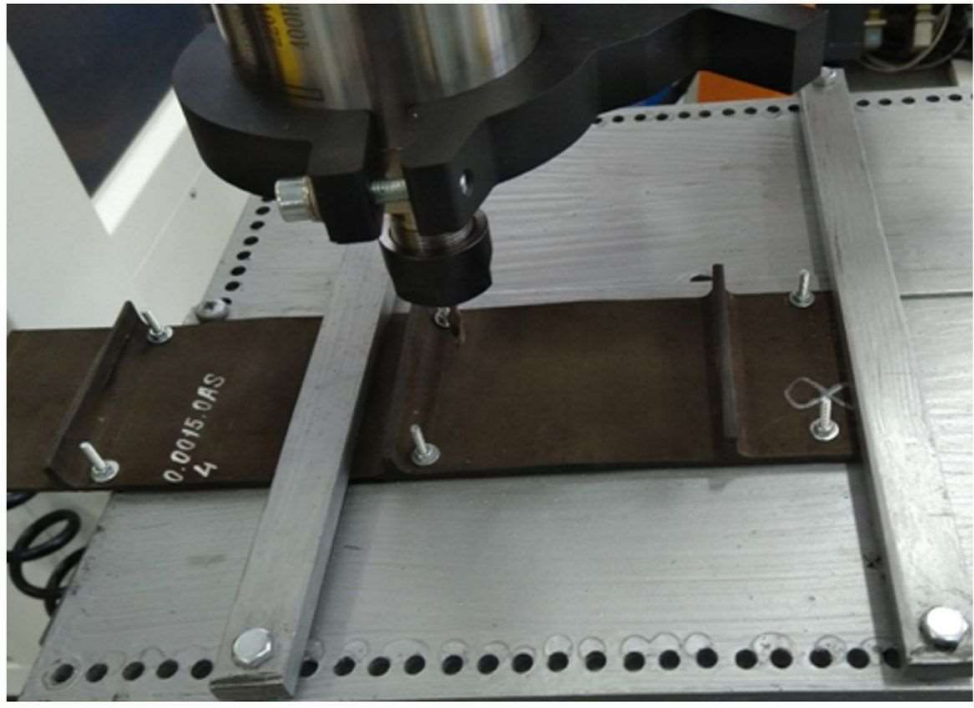

b)

Fig. 1. General view of the experimental setup to study the process of ultrasonic drilling: a- side view; $\mathrm{b}$ - top view of the machining.

In addition to the industrial robot, the installation contains a spindle attached to the robot; Ultrasonic head, dynamometer UDM-100 and a stage, on which a package of CM and titanium alloys is fixed.

For the generation of ultrasonic vibrations, an ultrasonic generator UZG-641A manufactured by NPP AVIASTEK with power Pout $=0.12 \mathrm{~kW}$ is used, which produces electrical signals with a frequency of $22 \mathrm{kHz}$. Further, the electrical signals, entering the 
ultrasonic head, are transformed by a piezoelectric transducer into mechanical vibrations of the same frequency and transmitted, depending on the execution of the assembled experimental setup, either to the spindle (version I) or to the complex package (version II) .

The spindle was a GDZ-80 milling spindle with a capacity of $2.2 \mathrm{~kW}(400 \mathrm{~Hz} ; 220 \mathrm{~V})$ with variable speed up to $24000 \mathrm{rpm}$, equipped with a set of ER20 collet chucks.

The authors used SER 108M DRILL Ø 4.7-0.03 mm DIN 6539 Ti-NAMITE-C COATED from SGS, recommended by the manufacturer for drilling titanium and aluminum alloy blanks, as well as non-metallic materials.

The device allows to carry out experiments with the imposition of unmodulated, amplitude-modulated or frequency-modulated ultrasonic vibrations on the drill or on the object table with a package of CM.

\section{Conclusion}

The results of preliminary experiments confirmed the positive influence of the energy of ultrasonic vibrations on the drilling process of complex packages consisting of $\mathrm{CM}$, titanium and (or) aluminum alloys.

\section{References}

1. Carvajal R, J. of Engineering Manufacture, 7 (2011)

2. Chakraborty D, Artificial neural network based delamination prediction in laminated composites. Materials and Design, 26 (2011)

3. DeVlieg R, One-Up Assembly with Robots, 2008-01-2297 (2010)

4. Díaz-Álvarez J, T. E. of Thermal Effects in Drilling of Woven Carbon Fiber Composite. Materials, 7 (2014)

5. Garrick, R., PCD Drills. SAE Technical, 3893 (2010)

6. Ghobadi A, Common Type of Damages in Composites and Their Inspections. W. J. of Mechanics, 2 (2017)

7. Antonialli Armando, Diniz Anselmo, Pederiva Robson, I. J. of Machine Tools and Manufacture, 50:65 (2010)

8. B. Burdo. Rus. Eng. R., 37:49 (2016)

9. Voronenko V, S. int. technologies in mechanical engineering, 45 (2018)

10. Ko, Aung, S. int. technologies in mechanical engineering, 21 (2019)

11. Xiao Gaobo, Ren Mingjun, To Suet, Micromachines. 9:49 (2018)

12. Srichand Hinduja, Yongsheng Ma, Gibbou Barrow, I. J. of Machine Tools and Manufacture 35:689 (2015)

13. M Bogoljubova, A Afonasov, V Kozlov and O Sumtsova, IOP Conf. Series: Materials Science and Engineering 124 (2016)

14. Alexey Popov, Sergei Babak, M. T. 18:1011 (2018)

15. Yohanes Waloyo, AIP Conf. Proceedings, 2097 (2019) 